\title{
LINC_00355 promotes gastric cancer progression by upregulating PHF19 expression through sponging miR-15a-5p
}

\author{
Jishui Zhang ${ }^{* *}$ D, Wenhao Lv', Yagang Liu', Weihua Fu², Baosheng Chen', Qiutong Ma and Xin Gao ${ }^{3}$
}

\begin{abstract}
Background: Long non-coding RNAs exert vital roles in several types of cancer. The objective of this study was to explore the role of LINC_00355 in gastric cancer (GC) progression and its potential mechanism.

Methods: The expression levels of LINC_00355 in GC tissues and cells were detected by quantitative real-time PCR, followed by assessing the effects of LINC_00355 knockdown or overexpression on cell properties. Dual-luciferase reporter assay was utilized to identify the relationship between LINC_00355 and microRNA (miR)-15a-5p and miR$15 a-5 p$ and PHD finger protein 19 (PHF19), followed by the rescue experiments.

Results: The results showed that LINC_00355 was highly expressed in GC tissues and cells compared with the corresponding control. LINC_00355 knockdown decreased the viability, migration, and invasion and increased the accumulation of GC cells in G1 phase and apoptosis. Meanwhile, LINC_00355 downregulation markedly increased cleaved caspase 3 and cleaved poly (ADP-ribose) polymerase protein levels, whereas decreased cyclin D1, cyclin E, matrix metalloproteinase (MMP) 9, MMP2, and N-cadherin protein levels in GC cells. However, LINC_00355 overexpression had the opposite effects. It was verified that LINC_00355 upregulated the expression of PHF19 through sponging miR-15a-5p. Furthermore, PHF19 overexpression reversed the effect of LINC_00355 knockdown on GC cell properties, including cell viability, migration, invasion, and apoptosis.
\end{abstract}

Conclusions: Collectively, these results suggest that LINC_00355 promotes GC progression by up-regulating PHF19 through sponging miR-15a-5p. Our findings may provide an important clinical basis for reversing the malignant phenotype of GC.

Keywords: Gastric cancer, LINC_00355, miR-15a-5p, PHD finger protein 19

\section{Background}

Gastric cancer (GC) is one of the most common cancers in the world and the third leading cause of cancer death [1]. According to statistics in 2018, there were 1,033,000 newly diagnosed GC cases and 783,000 deaths worldwide, indicating that $\mathrm{GC}$ has become a serious threat to human health [2]. In recent years, with the development

\footnotetext{
*Correspondence: jishui_z1980@163.com

'Department of Gastrointestinal Surgery, Cangzhou Central Hospital, No. 16 Xinhua West Road, Cangzhou 061001, Hebei, China

Full list of author information is available at the end of the article
}

of colonoscopy and surgical techniques, the 5-year mortality rate of early GC has been significantly reduced. However, for advanced GC, the 5-year mortality rate was still high [3]. Therefore, exploring the mechanism of GC development and potential therapeutic targets may provide a new idea for the treatment of GC.

Long noncoding RNA (lncRNA) is a non-coding RNA with a length of more than $200 \mathrm{nt}$. LncRNA can act as a tumor inducer or suppressor in the development of cancers $[4,5]$. It has been found that LINC_00355 was highly expressed in bladder transitional cell carcinoma

(c) The Author(s). 2021 Open Access This article is licensed under a Creative Commons Attribution 4.0 International License, which permits use, sharing, adaptation, distribution and reproduction in any medium or format, as long as you give appropriate credit to the original author(s) and the source, provide a link to the Creative Commons licence, and indicate if changes were made. The images or other third party material in this article are included in the article's Creative Commons licence, unless indicated otherwise in a credit line to the material. If material is not included in the article's Creative Commons licence and your intended use is not permitted by statutory regulation or exceeds the permitted use, you will need to obtain permission directly from the copyright holder. To view a copy of this licence, visit http://creativecommons.org/licenses/by/4.0/. The Creative Commons Public Domain Dedication waiver (http://creativecommons.org/publicdomain/zero/1.0/) applies to the data made available in this article, unless otherwise stated in a credit line to the data. 
[6]. LINC_00355 was involved in the regulation of competitive endogenous RNA (ceRNA) networks and was differentially expressed in multiple pathological stages of colorectal cancer [7]. Previous findings indicated a vital role of LINC_00355 in cancer development. However, whether LINC_00355 plays a role in the development of GC remains unknown.

There is increasing evidence that lncRNA plays the role of ceRNA. LncRNA can regulate the expression of target genes through competitively binding of microRNA (miRNA) $[8,9]$. The ceRNA hypothesis provides a new idea for the identification of lncRNA functions [10]. Wei et al. [11] found that LINC_00355 was highly expressed in lung adenocarcinoma, and LINC_00355 promoted the proliferation and colony formation of lung adenocarcinoma cell and inhibited cell cycle arrest and apoptosis through competitively binding to miR-195. Lu et al. [12] showed that LINC_00355 served as a miR-195 sponge to enhance viability, invasion, migration, and inhibit apoptosis of head and neck squamous cell carcinoma (HNSC C) cells by increasing homeoboxA10 expression. In present study, we focused on the role of lncRNA LINC_ 00355 in GC. We hypothesized that LINC_00355 might be involved in the development of $\mathrm{GC}$ via regulating miRNA/mRNA axis.

\section{Methods}

\section{Sample and clinical data collection}

GC tissues and adjacent tissues $(n=30)$ were collected from the Cangzhou Central Hospital. The patients did not receive any surgery, chemotherapy, radiation, or other anti-cancer treatment.

\section{Cell culture and transfection}

The GC cells lines (AGS, SNU-1, MKN45, HGC-27) were purchased from Procell Life Science\&Technology Co.,Ltd. (Wuhan, China), Fuheng Biotechnology Co., Ltd. (Shanghai, China), and Zhong Qiao Xin Zhou Biotechnology Co., Ltd. (Shanghai, China). HGC-27 cells were cultured in Roswell Park Memorial Institute (RPMI)-1640 medium (Hyclone, South Logan, UT, USA) supplemented with $20 \%$ fetal bovine serum (FBS, Hyclone). MKN45 cells were cultured in RPMI-1640 medium (Hyclone) supplemented with 10\% FBS (Hyclone). These cells were incubated in an incubator at $37^{\circ} \mathrm{C}$ with $5 \% \mathrm{CO}_{2}$.

HGC-27 and MKN45 cells were seeded in 6-well plates with a density of $4 \times 10^{5}$ cells/well and then cultured in an incubator at $37{ }^{\circ} \mathrm{C}$ with $5 \% \mathrm{CO}_{2}$. After $24 \mathrm{~h}$ incubation, cells were transfected with siRNA LINC_ 00355 (si-LINC_00355), pcDNA3.1-PHF19 plasmid or its negative control (si-NC/vector) for $24 \mathrm{~h}$. For the cotransfection, HGC-27 and MKN45 cells were cotransfected with si-LINC_00355 and pcDNA3.1-PHD finger protein 19 (PHF19) plasmid for $24 \mathrm{~h}$ or $48 \mathrm{~h}$. The transfections were mediated by Lipofectamine ${ }^{\text {nax }} 2000$ (Invitrogen, Carlsbad, CA, USA).

\section{RNA isolation and quantitative real-time PCR (RT-qPCR) analysis}

Total RNA were isolated from tissues and cells by using total RNA extraction kit (BioTeke, Beijing, China) according to the manufacturer's instructions. RNA was then reverse-transcribed to complementary DNA (cDNA) by using a reverse transcriptase kit (Takara, Dalian, China). RT-qPCR was conducted using cDNA, primers, SYBR Green, and Taq HS Perfect Mix. Primers are synthesized by GenScript Biotechnology Co., Ltd. (Nanjing, China). The $\beta$-actin was used as a loading control for LINC_00355 and PHF19. U6 served as a loading control for miR-15a-5p. The relative expression levels of genes were calculated by using $2^{-\Delta \Delta c t}$ analysis.

\section{Cell viability assay}

Cell viability was measured by Cell Counting Kit- 8 assay. Cells were seeded in 96-well plates with a density of $3 \times$ $10^{3}$ cells/well. After transfection, cells were placed in an incubator for $0,6,24,48$, and $72 \mathrm{~h}$, respectively, at $37^{\circ} \mathrm{C}$ with $5 \% \mathrm{CO}_{2}$. Subsequently, CCK-8 $(10 \mu \mathrm{l})$ was added to each well and incubated at $37^{\circ} \mathrm{C}$ in $5 \% \mathrm{CO}_{2}$ for $1 \mathrm{~h}$. For each well, the optical density value was measured at a wavelength of $450 \mathrm{~nm}$.

\section{Cell apoptosis analysis}

Cells were placed in 6-well plates with a density of $4 \times$ $10^{5}$ cells/well. After transfection, cells were harvested and treated with AnnexinV-FITC $(5 \mu \mathrm{l})$ and propidium iodide (PI, $10 \mu \mathrm{l})$. After incubation at room temperature in the dark for $15 \mathrm{~min}$, cells were resuspended in $1 \times$ binding buffer. The percentage of apoptotic cells was calculated by using flow cytometry (Aceabio, San Diego, CA, USA).

\section{Cell cycle analysis}

Cells were seeded into 6-well plates at a density of $4 \times$ $10^{5} /$ well. After transfection, cells were harvested and fixed in ethanol $(70 \%)$ at $4{ }^{\circ} \mathrm{C}$ for $12 \mathrm{~h}$. Subsequently, the cells were collected by centrifugation and resuspended in staining buffer and incubated with PI staining solution $(25 \mu \mathrm{l})$ and RNase A $(10 \mu \mathrm{l})$ in the dark for $30 \mathrm{~min}$. Flow cytometry (Aceabio) was used to detect cell cycle.

\section{Western blot analysis}

Total protein was extracted from cells and separated with SDS polyacrylamide gel electrophoresis. The proteins were then transferred onto the polyvinylidene difluoride (PVDF) membranes. The membranes were incubated with anti-cyclin D1 (1:1000, Cell Signaling 
Technology (CST), Danvers, MA, USA), anti-cyclin E (1: 500, Affinity Biosciences, Cincinnati, OH, USA), antipro/cleaved caspase 3 (1:500, CST), anti-pro/cleaved poly (ADP-ribose) polymerase (PARP) (1:1000, CST), anti-matrix metalloproteinase (MMP) 2 (1:1000, CST), anti-MMP9 (1:1000, CST), anti-N-cadherin (1:500, CST), anti-PHF19 (1:500, Affinity Biosciences), and anti$\beta$-actin (1:2000, Proteintech, Wuhan, China) at $4{ }^{\circ} \mathrm{C}$ overnight. After washing three times $(5 \mathrm{~min} /$ time $)$ with TBST, the membranes were incubated with horseradish peroxidase-conjugated goat anti-rabbit/mouse IgG (1: 10000, Proteintech) for $2 \mathrm{~h}$. After washing three times (5 $\mathrm{min} /$ time) with TBST, the membranes were visualized by treated with electrochemiluminescence luminescent solution. $\beta$-actin served as a loading control for protein expression.

\section{Cell migration assay}

Cell migration was assessed by wound-healing assay. The cells of each group were cultured to the density of fusion state. Before the experiment, the medium was changed to serum-free medium and treated with mitomycin C $(1 \mu \mathrm{g} / \mathrm{ml})$ for $1 \mathrm{~h}$. The cells were scratched with $200 \mu \mathrm{l}$ pipette tip and the surface of the cells was washed once with serum-free medium to remove cell debris. Cells were then incubated in an incubator at $37^{\circ} \mathrm{C}$ with $5 \% \mathrm{CO}_{2}$ for $0 \mathrm{~h}$ and $24 \mathrm{~h}$, respectively, and then photographed, and the location of the cells was recorded.

\section{Cell invasion assay}

Cell invasion was assessed by transwell assay. Transwell chamber coated with Matrigel gel was placed into a 24well plate. Culture medium ( $800 \mu \mathrm{l})$ containing 10\% FBS was added to the lower chamber and cell suspension $(200 \mu \mathrm{l})$ was added to the upper chamber $\left(2 \times 10^{4}\right.$ cells/ well). The 24-well plates were cultured in a cell incubator at $37{ }^{\circ} \mathrm{C}$ with $5 \% \mathrm{CO}_{2}$. Subsequently, after fixed in $4 \%$ paraformaldehyde at room temperature for $25 \mathrm{~min}$, the cells were stained with $0.4 \%$ crystal violet staining solution for $5 \mathrm{~min}$ and rinsed with distilled water. Cells migrating to the sublayer of the microporous membrane were counted under an inverted microscope (Olympus, Tokyo, Japan).

\section{Dual-luciferase reporter assay}

The binding sites of LINC_00355 and miR-15a-5p were predicted by bioinformatics analysis. (LncBase v.2, http://carolina.imis.athena-innovation.gr/diana_tools/ web/index.php? $r=$ Incbasev2/index-predicted). The binding sites of miR-15a-5p and PHF19 were predicted by TargetScanHuman 7.2 (http://www.targetscan.org/vert $72 /$ ). The wild type (wt) and mutant (mut) fragments of LINC_00355 were cloned into pGLO vector (GenScript, Nanjing, China) to construct LINC_00355-wt and
LINC_00355-mut plasmids. The 3'-untranslated region (3'-UTR) sequences of PHF19 containing predicated or mutated miR-15a-5p binding sites were used to construct PHF19 3'-UTR-wt and PHF19 3'-UTR-mut plasmids. Cells were co-transfected with miR-15a-5p mimic (12.5 or 25 pmol per well) or its NC (12.5 or $25 \mathrm{pmol}$ per well) and corresponding reporter plasmids $(0.5 \mu \mathrm{g}$ per well) using Lipofectamine ${ }^{\mathrm{Tm}} 2000$ (Invitrogen). At 48 $\mathrm{h}$ after transfection, luciferase assay kit (KeyGEN, Jiangsu, China) was used to detect luciferase activity.

\section{Statistical analysis}

All data analysis was conducted using GraphPad version 8.0. Comparisons between groups were estimated by the paired t-test, one-way ANOVA or two-way ANOVA. $P<0.05$ was considered statistically significant.

\section{Results}

\section{LINC_00355 was highly expressed in GC tissues and cell lines}

We first explored the expression level of LINC_00355 in GC tissues and cells by RT-qPCR. As shown in Fig. 1a, the expression of LINC_00355 in 30 pairs of GC tissues was significantly higher than that of adjacent tissues $(P=0.0123)$. Meanwhile, the LINC_00355 expression levels in GC cells lines (AGS, MKN45, and HGC-27) were significantly increased as compared with normal gastric cells (GES-1) (Fig. 1b). Among them, MKN45 and HGC-27 cell lines with relatively high expression levels of LINC_00355 were used for subsequent experiments. Overall, the results indicated that LINC_00355 may be positively associated with the development of GC.

\section{LINC_00355 silencing inhibited the proliferation and promoted apoptosis in GC cells}

To investigate the role of LINC_00355 in GC cell proliferation and apoptosis, we knocked down the expression of LINC_00355 by transfecting siRNA. The results showed that si-LINC_00355 transfection significantly decreased LINC_00355 expression compared with control group in HGC-27 and MKN45 cells (Fig. 2a). The results of CCK-8 detection showed that LINC_00355 silencing observably inhibited the viability in GC cells, whereas LINC_00355 overexpression increased the viability of GC cells (Fig. 2b and SF. 1c). The cell cycle distribution and apoptosis rate was detected by flow cytometry, and the results showed that LINC_00355 knockdown led to the accumulation of GC cells in the G1 phase and induced apoptosis (Fig. 2c and d). Meanwhile, the protein expression levels of apoptosis-related factors cleaved caspase 3 and cleaved PARP was markedly increased by LINC_00355 downregulation (Fig. 2e). The si-LINC_ 00355 transfection significantly reduced cyclin D1 and 


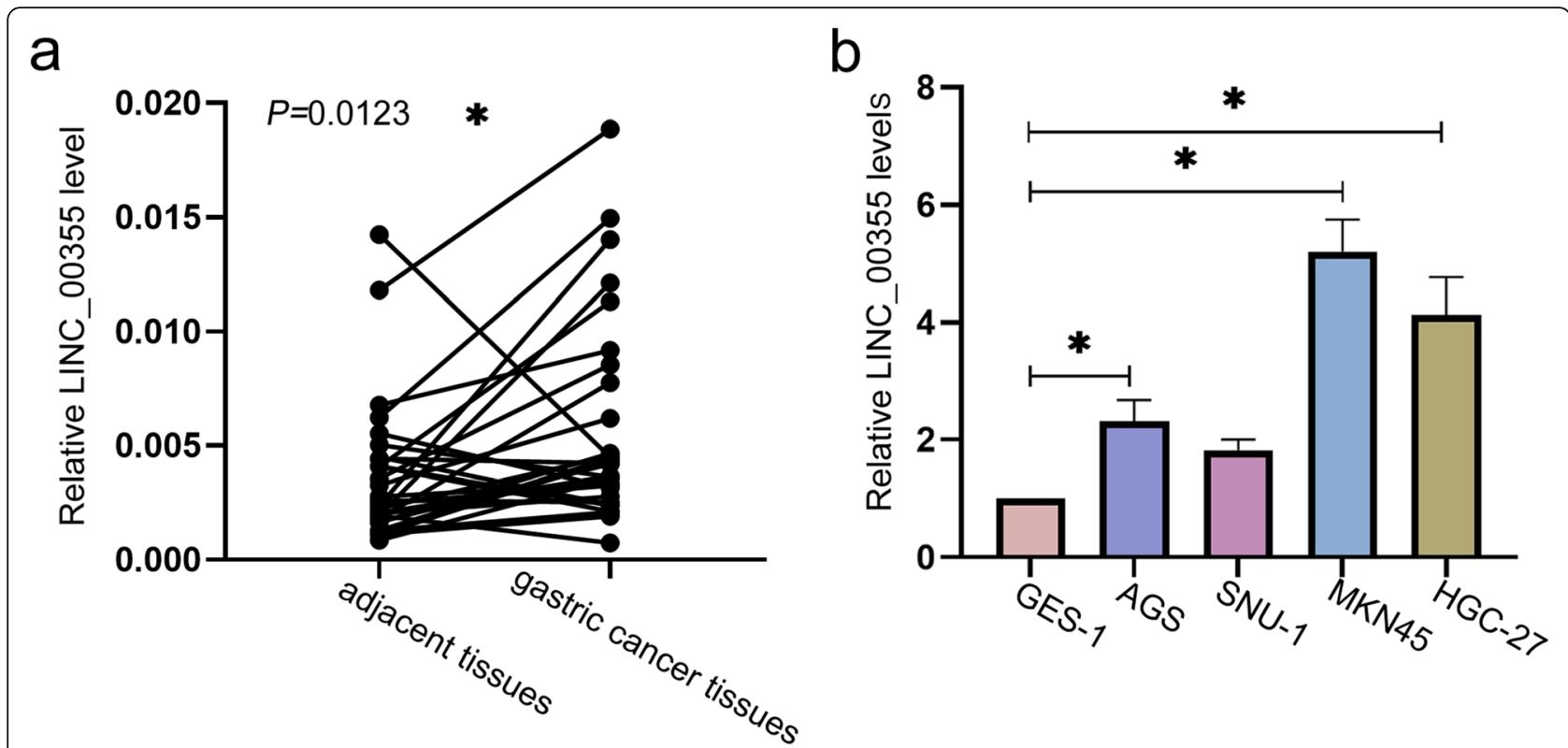

Fig. 1 LINC_00355 was highly expressed in GC tissues and cell lines. a The expression levels of LINC_00355 in GC tissues and adjacent tissues $(n=30)$ detected by quantitative real-time PCR. $P<0.05$ compared with adjacent tissues. b Relative LINC_00355 expression levels in GC cells lines (AGS, SNU-1, MKN45, HGC-27) and normal gastric cells (GES-1). All values were expressed as mean \pm standard deviation. $n=3$. ${ }^{*} P<0.05$ compared with GES-1. GC, gastric cancer

cyclin $\mathrm{E}$ protein levels in comparison with the si-NC group (Fig. 2e). Conversely, the protein level of cyclin D1 in GC cells was significantly increased by LINC_ 00355 overexpression (SF. 1f). Overall, the results indicated that LINC_00355 can promote cell viability and inhibit apoptosis during GC progression.

\section{LINC_00355 silencing suppressed the migration and invasion in GC cells}

We further explored the effects of LINC 00355 knockdown on migration and invasion of GC cells. As presented in Fig. 3a, the migration and invasion ability of GC cells with LINC_00355 knockdown was lower than that in the si-NC group, whereas LINC_ 00355 upregulation showed the opposite effects (Fig. $3 \mathrm{a}$ and $\mathrm{b}$ and SF. 1d and e). Additionally, the protein expression levels of MMP9, MMP2, and N-cadherin were markedly decreased in si-LINC_00355-1 and si-LINC_00355-2 groups in comparison with the siNC group (Fig. 3c). However, the MMP2 and MMP9 protein levels in LINC_00355-overexpressed cells were markedly increased (SF. 1f). Thus, these results demonstrate that LINC_00355 is positively correlated with the migration and invasion of GC cells.

\section{LINC_00355 up-regulated PHF19 expression through sponging miR-15a-5p in GC cells}

We further explored the specific mechanism of LINC_00355 involved in the development of GC. Bioinformatics analysis predicted the binding sites between LINC_00355 and miR-15a-5p (Fig. 4a). Moreover, the results of dual-luciferase reporter assay indicated that the luciferase activity in LINC_ 00355 wt was significantly decreased by miR-15a-5p mimic in a dose-dependent manner and no significant changes was observed in other groups (Fig. 4a and SF. 2). The expression level of miR-15a-5p in GC cells was detected by RT-qPCR and showed that the depletion of LINC_00355 significantly increased miR-15a-5p expression levels in HGC-27 and MKN45 cells (Fig. 4b). On the contrary, the protein expression levels of PHF19 in HGC-27 and MKN45 cells were decreased by LINC_00355 downregulation (Fig. 4c). Bioinformatics analysis predicted the binding sites between miR-15a-5p and PHF19 (Fig. 4d). Luciferase assays indicated that the luciferase activities of PHF19 3'-UTR-wt were decreased by miR15a-5p mimic dose-dependently (Fig. 4d and SF. 2). Results of RT-qPCR analysis showed that the expression level of miR-15a-5p was significantly increased by miR-15a-5p mimic and decreased by miR-15a-5p inhibitor in $\mathrm{HGC}-27$ and MKN45 cells (Fig. 4e). However, the protein level of PHF19 was markedly decreased by miR-15a-5p overexpression and increased by miR-15a-5p knockdown (Fig. 4f). Collectively, the results indicate that LINC_00355 acts as a ceRNA to regulate PHF19 expression via competitively binding to miR-15a-5p. 


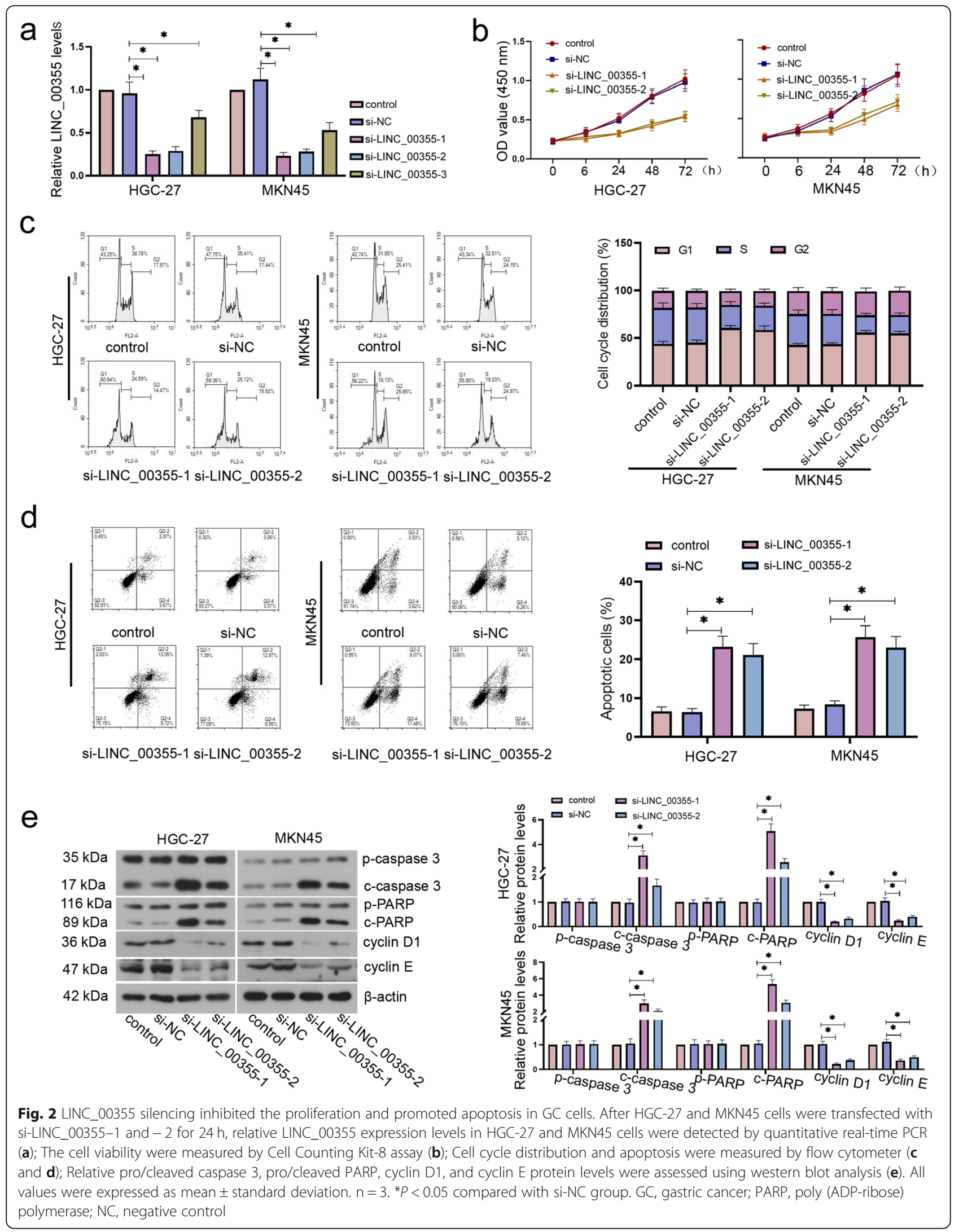




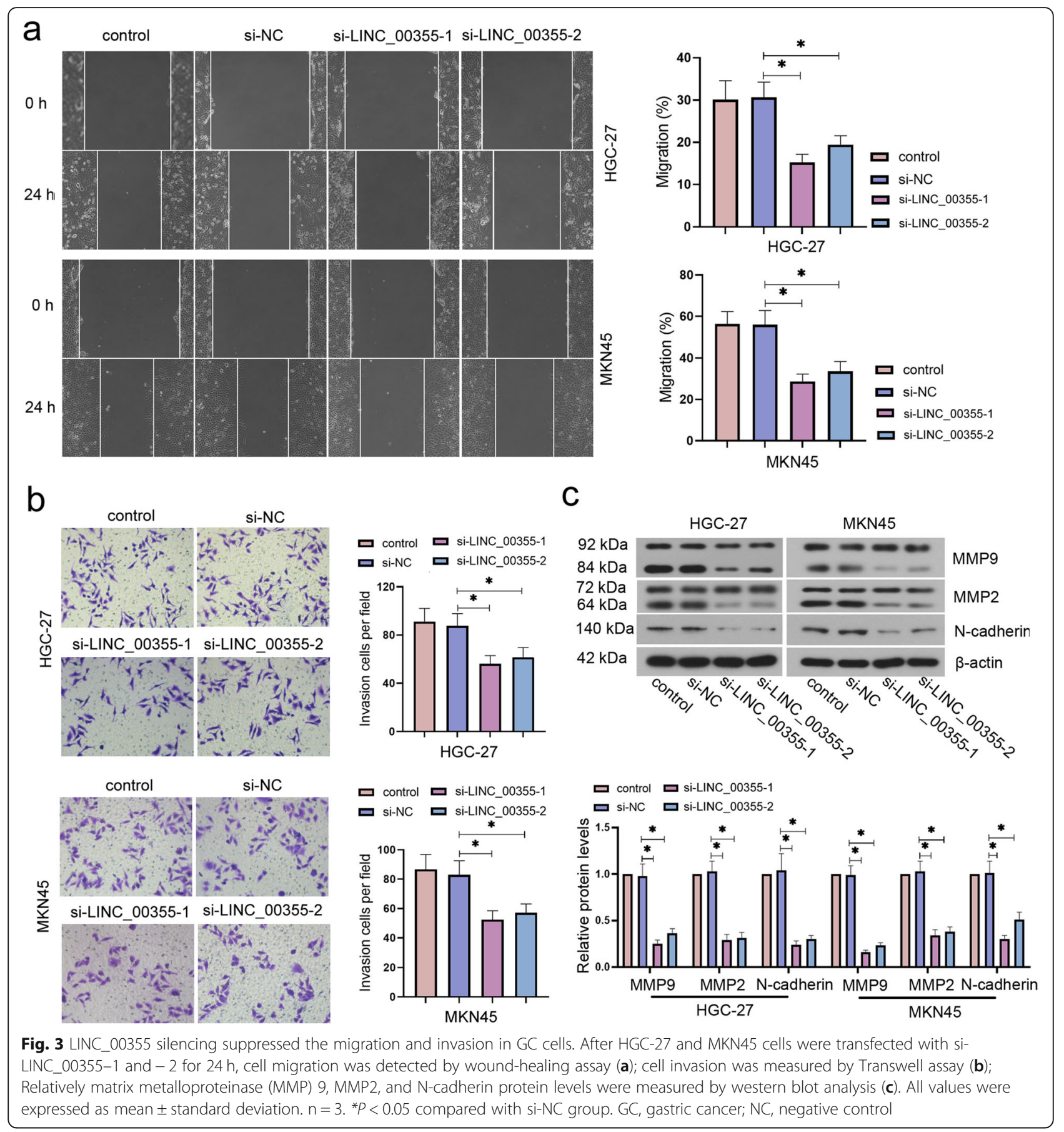

PHF19 overexpression reversed the effect of LINC_00355 knockdown on the development of GC

We next analyzed the correlation between LINC_00355 and PHF19 expression via Pearson correlation analysis. The results showed that the expression of PHF19 in GC tissues $(n=30)$ was positively correlated with LINC_ 00355 (SF. $1 \mathrm{~g}$ ). To verify whether LINC_00355 plays a role in GC progression through regulating PHF19 expression, HGC-27 and MKN45 cells were co-transfected with si-LINC_00355 and pcDNA3.1-PHF19 plasmid. As shown in SF. 1a, the transfection efficiency of pcDNA3.1-PHF19 plasmid was first verified in HGC-27 and MKN45 cells. PHF19 overexpression significantly increased viability, invasion, and migration and inhibited apoptosis in GC cells in comparison with the si-NC+ vector group (Fig. 5a-d). Moreover, PHF19 overexpression reversed the effect of LINC_00355 knockdown on GC cell functions, including cell viability, migration, 
a

LINC_00355 wt: 5'...AGGAUUAUAAAUCAUGCUGCUA.. miR-15a-5p: 3'...GUGUUUGGUAAUACACGACGAU...5' LINC_00355 mut: 5'...AGGAUUAUAAAUCAACGACGAA...3’

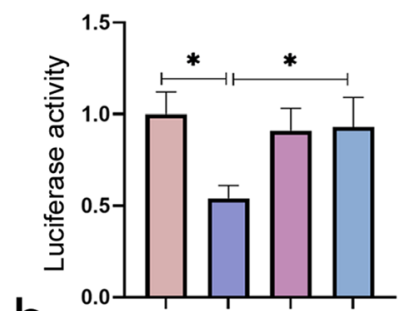

miR-15a-5p mimic: 25 pmol/well mimic NC: $25 \mathrm{pmol} / \mathrm{well}$ 口LINC_00355 wt+mimic NC 口LINC_00355 wt+miR-15a-5p mimic 口LINC_00355 mut+mimic NC - LINC_00355 mut+ miR-15a-5p mimic

b

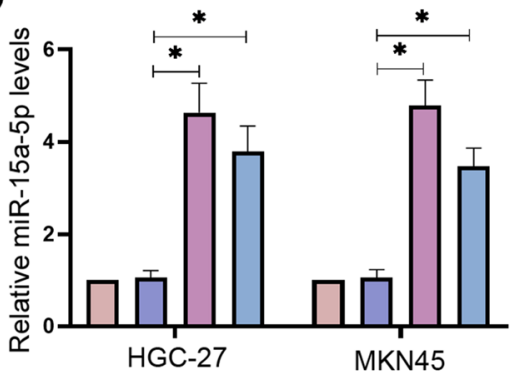

C
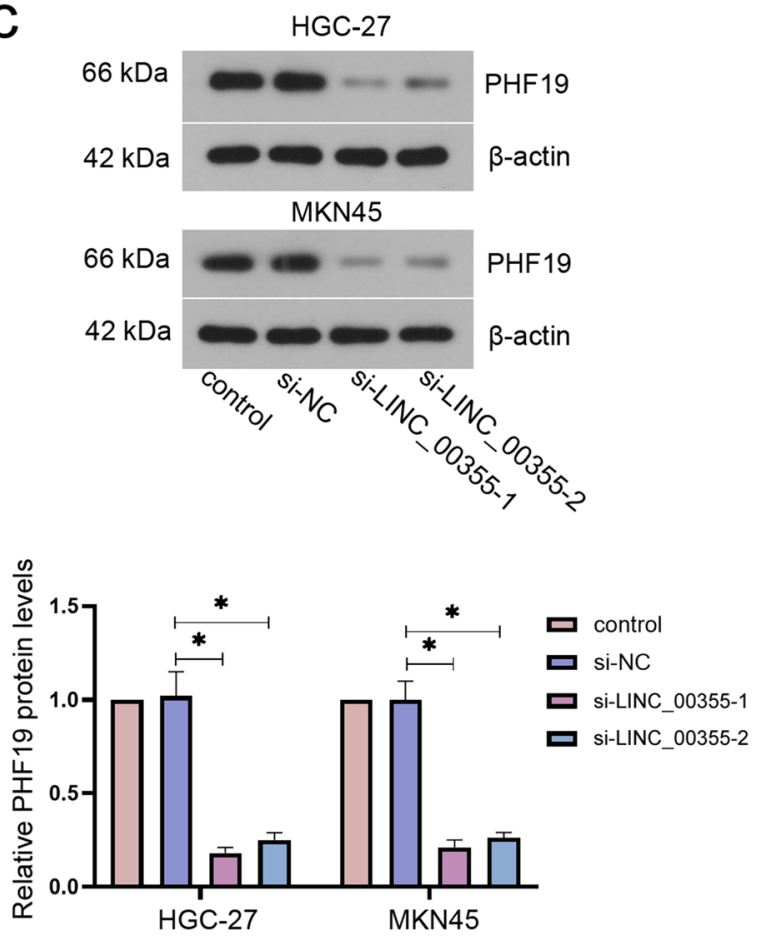

d

PHF19 wt: $\quad$ ',...GACCCCUGAUGUUUUGCUGCUU..3' miR-15a-5p: 3'...GUGUUUGGUAAUACACGACGAU...5' PHF19 mut: $\quad 5^{\prime}$...GACCCCUGAUGUUUACGACGAU...3'

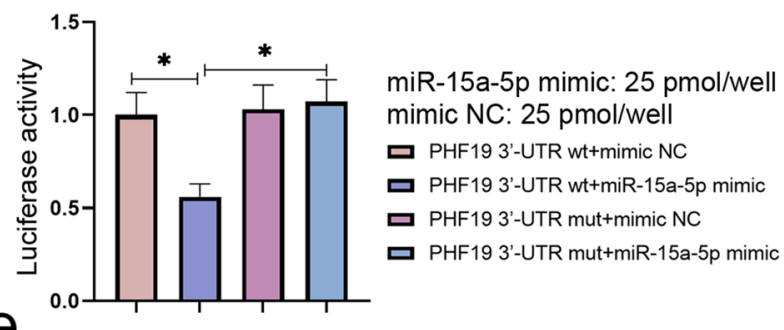

e

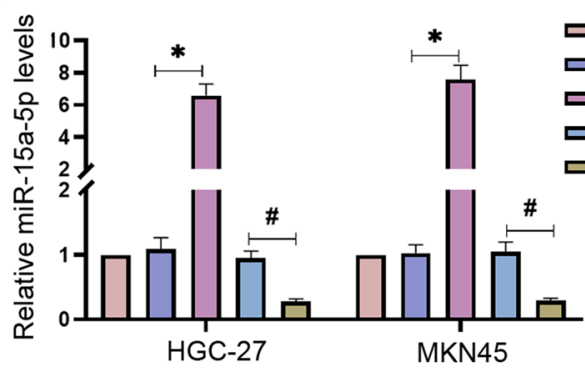

f

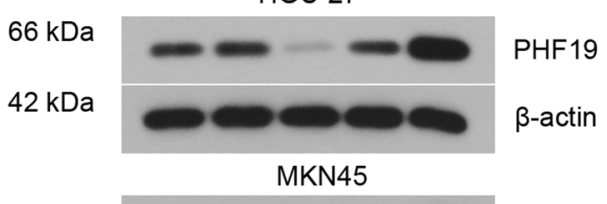

$66 \mathrm{kDa}$

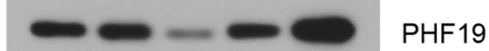

$42 \mathrm{kDa}$

$\beta$-actin
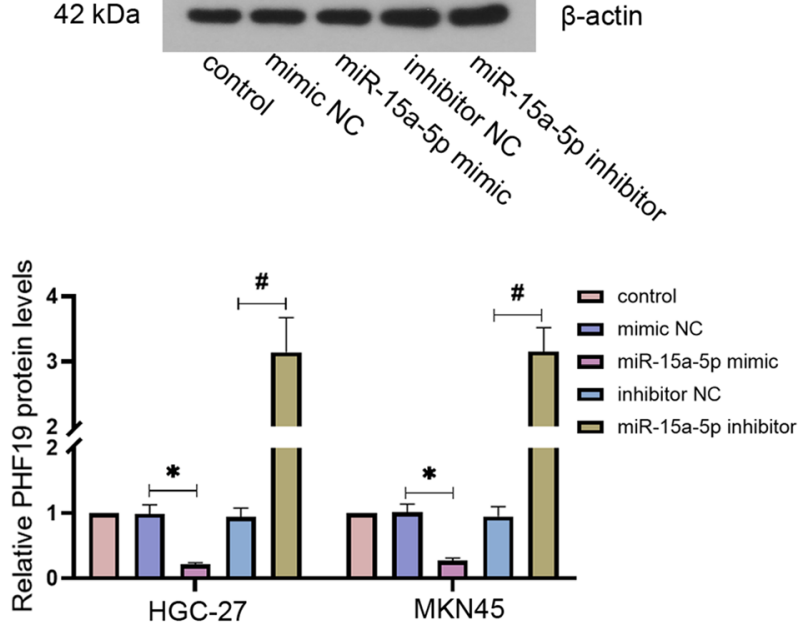

Fig. 4 (See legend on next page.) 
(See figure on previous page.)

Fig. 4 LINC_00355 upregulated PHF19 expression through sponging miR-15a-5p in GC cells. a Relative luciferase activity in 293 T cells cotransfected with LINC_00355 reporter plasmid and the candidate miRNA. ${ }^{*} P<0.05$ compared with LINC_00355 wt + miR-15a-5p mimic group (b) Relative miR-15a-5p expression levels in HGC-27 and MKN45 cells transfected with si-LINC_00355-1 and - 2. ${ }^{*} P<0.05$ compared with si-NC group. c Relative PHF19 protein expression levels in HGC-27 and MKN45 cells transfected with si-LINC_00355-1 and -2 . ${ }^{*} P<0.05$ compared with si-NC group. $\mathbf{d}$ Relative luciferase activity in 293 T cells co-transfected with PHF19 reporter plasmid and the candidate miRNA. ${ }^{*} P<0.05$ compared with PHF19 3'-UTR wt + miR-15a-5p mimic group. e The expression levels of miR-15a-5p in HGC-27 and MKN45 cells transfected with miR-15a-5p mimic, inhibitor, or its corresponding negative control. ${ }^{*} P<0.05$ compared with mimic NC group and ${ }^{\#} P<0.05$ compared with inhibitor NC group. $\mathbf{f}$ Relative PHF19 protein levels in HGC-27 and MKN45 cells transfected with miR-15a-5p mimic or inhibitor. * $P<0.05$ compared with mimic NC group and ${ }^{\#} P<0.05$ compared with inhibitor NC group. All values were expressed as mean \pm standard deviation. $n=3$. GC, gastric cancer; $N C$, negative control

invasion, and apoptosis (Fig. 5a-d). Additionally, PHF19 upregulation markedly decreased the protein expression levels of cleaved caspase 3 and cleaved PARP, whereas significantly increased cyclin D1, cyclin E, MMP9, and MMP2 protein levels as compared with si-NC + vector group in HGC-27 and MKN45 cells (Fig. 5e). Furthermore, PHF19 upregulation inverted the effect of LINC 00355 silencing on the protein expression levels of cleaved caspase 3, cleaved PARP, cyclin D1, cyclin E, MMP9, and MMP2 (Fig. 5e). Overall, these results indicate that LINC_00355 may promote the development of GC by sponging miR-15a-5p to regulate PHF19 expression.

\section{Discussion}

This study was the first to investigate the role of LINC_ 00355 in the development of GC and its potential mechanisms. We demonstrated that LINC_00355 was highly expressed in GC tissues and cells. LINC_00355 knockdown inhibited the proliferation, migration, and invasion and induced apoptosis of GC cells. Furthermore, LINC_ 00355 increased the expression of PHF19 by competitively inhibiting its binding to miR-15a-5p. Moreover, PHF19 was identified as a facilitator of GC development. Therefore, the present study demonstrated that LINC 00355 may promote the development of GC by upregulating miR-15a-5p-mediated PHF19 expression.

Various studies have shown that lncRNA plays a vital role in regulating cell processes, including cell cycle, growth, and apoptosis $[13,14]$. LncRNA changes at the transcriptional or post-transcriptional levels directly or indirectly lead to uncontrolled tumor growth $[15,16]$. It was previously reported that LINC_00355 was associated with the clinical features of colorectal cancer and negatively correlates with survival rate [7]. Additionally, LINC_00355 was highly expressed in prostate cancer and associated with the survival rates of cancer patients [17]. Here, highly expressed LINC_00355 was also observed in GC tissues and cells, indicating that LINC_00355 may play a promoting role in the development of GC. Further studies indicated that downregulation of LINC_00355 suppressed the viability, invasion, migration, and promoted apoptosis of GC cells. LINC_00355 inhibition also improves the malignant phenotype of other cancers. For example, LINC_00355 knockdown inhibited the proliferation and invasion of bladder cancer cells [18]. The downregulation of LINC_00355 inhibited the viability, invasion, migration, and epithelial-mesenchymal transition and promoted apoptosis of cancer stem cells in HNSCC [12]. Collectively, LINC_00355 may participate in the development of $\mathrm{GC}$ by regulating the functions of GC cells, including proliferation, migration, invasion, and apoptosis.

More and more evidence supported the ceRNA hypothesis and showed that the ceRNA regulatory network was closely related to the development of GC. Bioinformatics analysis showed that there were binding sites between LINC_00355 and miR-15a-5p, which was verified by dualluciferase reporter assay. Notably, a single lncRNA often plays a role in cancer by sponging multiple miRNAs. For instance, LINC_00355 promoted HNSCC progression through binding miR-195 [12]. In hepatocellular carcinoma (HCC), LINC_00355 acted as a ceRNA to sponge miR-6777-3p and further promoted HCC progression [19]. These findings indicate that LINC_00355 regulates cancer development in multiple pathways and highlights the important role of LINC_00355 in cancer progression. In present study, we demonstrate that LINC_00355 promotes GC progression through sponging miR-15a-5p, indicating that the LINC_00355/miR-15a-5p axis may be one of the ways that LINC_00355 regulates the development of GC.

miR-15a-5p has been reported to play a negative regulatory role in the development of several cancers, including GC [20], bladder cancer [21], and cervical cancer [22]. Specifically, miR-15a-5p expression in GC patients was significantly decreased and miR-15a-5p overexpression suppressed GC cell proliferation and tumor invasion [23]. Zare et al. [24] demonstrated that miR-15a-5p downregulation was associated with advanced tumor grading and metastasis. miRNA is considered to regulate gene expression by binding to the 3 '-untranslated region ( 3 '-UTR) of the target genes and thus has a wide range of biological functions [25-27]. For instance, miR-15a-5p deletion suppressed the GC cell proliferation, monolayer colony formation, invasion and migration, and xenograft formation in vivo by targeting yes-associated protein 1 [20]. Here, we 


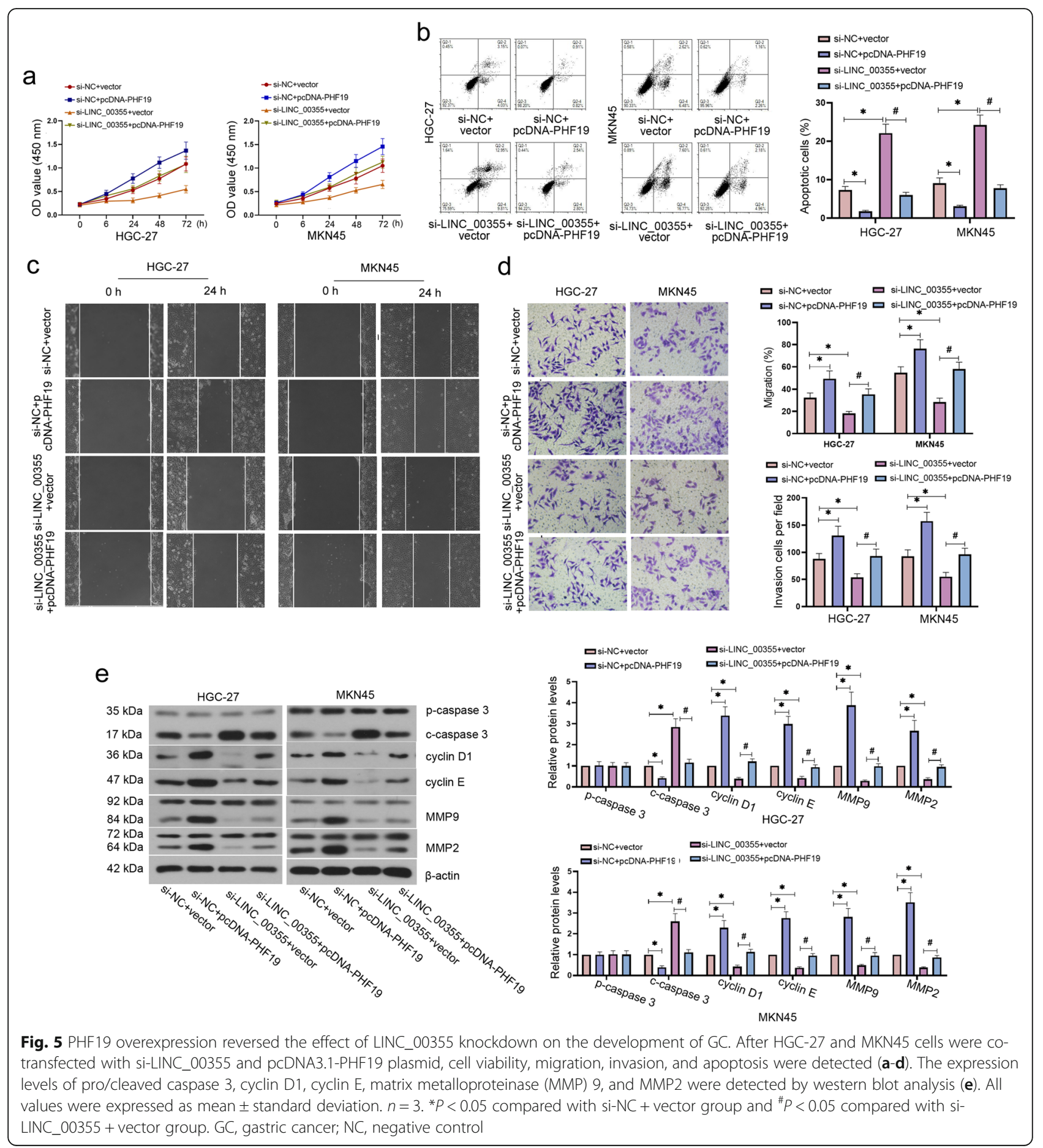

confirmed that PHF19 is one of the downstream targets of miR-15a-5p. PHF19 is a member of polycomblike proteins and can regulate the enzymatic activity and recruitment of polycomb inhibition complex 2 (PRC2) [28, 29]. The dysregulation of PHF19 expression is associated with the development of several cancers, including GC [30]. Previous studies have found a positive correlation between PHF19 expression and glioblastoma progression [31]. PHF19 silencing reduced the proliferation, and induced apoptosis and cell cycle stagnation in ovarian cancer cells [32]. Moreover, the overexpression of PHF19 was associated with the paclitaxel resistance of GC patients [33]. Additionally, a recent study have indicated that the downregulation of PHF19 could suppress the proliferation and migration of GC cells [30]. The findings highlight the positive regulatory role of PHF19 in cancer development. 
The present study also confirmed the promotion effects of PHF19 on GC progression. However, it is worth noting that a miRNA has multiple targets. This property enables miRNAs to have multiple functions through targeting different genes and suggests that miRNAs may be involved in the development of diseases, including cancer, through a variety of regulatory directions. This feature also highlights the important role of miRNAs in the pathological process. In present study, miR-15a-5p/PHF19 axis may be one of the ways that miR-15a-5p regulates the development of GC. Furthermore, considering the targeting relationship between LINC_00355 and miR-15a-5p, and miR15a-5p and PHF19, we demonstrated that LINC_00355 may upregulate the expression of PHF19 in GC through sponging miR-15a-5p. In addition, PHF19 overexpression restored the effect of LINC_00355 knockdown on GC progression, indicating that LINC_00355 may participate in the development of GC by upregulating PHF19 expression through sponging miR-15a-5p. Our findings suggest that LINC_00355/miR-15a-5p/PHF19 axis may be one of the ways that LINC_00355 regulates the development of GC.

\section{Conclusions}

The present study demonstrated that LINC_00355 was highly expressed in GC tissues and cells and promoted gastric cancer progression. This promotion was achieved by sponging miR-15a-5p to regulate PHF19 expression. Our findings may provide an important clinical basis for reversing the malignant phenotype of GC.

\section{Abbreviations}

GC: Gastric cancer; PHF19: PHD finger protein 19; MMP9: Matrix metalloproteinase 9; LncRNA: Long noncoding RNA; ceRNA: Competitive endogenous RNA; miRNA: microRNA; CDNA: Complementary DNA; PI: Propidium iodide; PARP: Poly (ADP-ribose) polymerase; Wt: Wild type; Mut: Mutant; 3'-UTR: 3'-untranslated region

\section{Supplementary Information}

The online version contains supplementary material available at https://doi. org/10.1186/s12885-021-08227-3.

Additional file 1: Supplementary Figure 1. (a) After HGC-27 and MKN45 cells were transfected with pcDNA3.1-PHF19 plasmid or its control for $24 \mathrm{~h}$, relative PHF19 mRNA expression levels were detected by quantitative real-time PCR. After HGC-27 cells were transfected with PCDNA3.1-LINC_00355 or empty vector for $24 \mathrm{~h}$, the transfection efficiency was detected by quantitative real-time PCR (b), the viability of HGC-27 cells was measured by CCK-8 (c), cell migration was assessed by wound-healing assay (d), cell invasion was measured by Transwell assay (e), relative protein levels of cyclin D1, MMP9, and MMP2 were detected by Western blot $(\mathrm{f})$. Correlation analysis of the expression levels of LINC_00355 and PHF19 in gastric cancer tissues (g). All values were expressed as mean \pm standard deviation. $n=3$.

Additional file 2: Supplementary Figure 2. Relative luciferase activity were measured using Dual-luciferase reporter assay in 293 T cells cotransfected with LINC_00355 reporter plasmid or PHF19 3'-UTR-wt/mut plasmid (12.5 pmol/well) and the candidate miRNA. All values were expressed as mean \pm standard deviation. $n=3$.
Additional file 3: Supplementary Figure 3. Original data of western blot.

\section{Acknowledgments}

Not applicable.

\section{Authors' contributions}

JSZ designed the study and wrote the manuscript. WHL, YGL, WHF, and BSC performed the experiments and analyzed the data. QTM and XG assisted with the performance of some experiments. All authors have approved the final manuscript.

\section{Funding}

This work was supported by a grant from the Medical Science Research Program of Health Commission of Hebei Province (No. 20200174). The funder had no role in the design of the study; collection, analysis, and interpretation of the data; or writing of the manuscript.

\section{Availability of data and materials}

The data supporting the conclusions of this article are included in the article (SF. 3).

\section{Declarations}

Ethics approval and consent to participate

All patients were informed about the use of the samples and signed informed consent. This study followed the institutional ethics guidelines, which were reviewed and approved by the Research Ethics Committee of Cangzhou Central Hospital.

Consent for publication

Not applicable.

\section{Competing interests}

The authors declare no competing interests.

\section{Author details}

${ }^{1}$ Department of Gastrointestinal Surgery, Cangzhou Central Hospital, No. 16 Xinhua West Road, Cangzhou 061001, Hebei, China. 'Department of General Surgery, Tianjin Medical University General Hospital, Tianjin 300052, China. ${ }^{3}$ Department of Radiotherapy, Cangzhou Central Hospital, Cangzhou 061001, Hebei, China.

Received: 5 November 2020 Accepted: 20 April 2021

Published online: 02 June 2021

References

1. Siegel R, Naishadham D, Jemal A. Cancer statistics, 2013. CA Cancer J Clin. 2013;63(1):11-30. https://doi.org/10.3322/caac.21166 PMID: 23335087.

2. Bray F, Ferlay J, Soerjomataram I, Siegel RL, Torre LA, Jemal A. Global cancer statistics 2018: GLOBOCAN estimates of incidence and mortality worldwide for 36 cancers in 185 countries. CA Cancer J Clin. 2018;68(6):394-424. https://doi.org/10.3322/caac.21492 PMID: 30207593.

3. Hamashima C, Shabana M, Okada K, Okamoto M, Osaki Y. Mortality reduction from gastric cancer by endoscopic and radiographic screening. Cancer Sci. 2015;106(12):1744-9. https://doi.org/10.1111/cas.12829 PMID: 26432528.

4. Dong Y, Wang ZG, Chi TS. Long noncoding RNA Lnc01614 promotes the occurrence and development of gastric cancer by activating EMT pathway. Eur Rev Med Pharmacol Sci. 2018;22(5):1307-14. https://doi.org/10.26355/ eurrev_201803_14472 PMID: 29565488.

5. Yan J, Zhang Y, She Q, Li X, Peng L, Wang X, et al. Long noncoding RNA H19/miR-675 Axis promotes Gastric Cancer via FADD/Caspase 8/Caspase 3 signaling pathway. Cell Physiol Biochem. 2017;42(6):2364-76. https://doi. org/10.1159/000480028 PMID: 28848149.

6. Yazarlou F, Modarressi MH, Mowla SJ, Oskooei VK, Motevaseli E, Tooli LF, et al. Urinary exosomal expression of long non-coding RNAs as diagnostic marker in bladder cancer. Cancer Manag Res. 2018;10:6357-65. https://doi. org/10.2147/CMAR.S186108 PMID: 30568497. 
7. Yuan W, Li X, Liu L, Wei C, Sun D, Peng S, et al. Comprehensive analysis of IncRNA-associated ceRNA network in colorectal cancer. Biochem Biophys Res Commun. 2019;508(2):374-9. https://doi.org/10.1016/j.bbrc.2018.11.151 PMID: 30503344.

8. Chan JJ, Tay Y. Noncoding RNA:RNA Regulatory Networks in Cancer. Int J Mol Sci. 2018;19(5). https://doi.org/10.3390/ijms19051310 PMID: 29702599.

9. Mao Y, Liu R, Zhou H, Yin S, Zhao Q, Ding X, et al. Transcriptome analysis of miRNA-IncRNA-mRNA interactions in the malignant transformation process of gastric cancer initiation. Cancer Gene Ther. 2017;24(6):267-75. https://doi. org/10.1038/cgt.2017.14 PMID: 28524153.

10. Thomson DW, Dinger ME. Endogenous microRNA sponges: evidence and controversy. Nat Rev Genet. 2016;17(5):272-83. https://doi.org/10.1038/nrg.2 016.20 PMID: 27040487.

11. Liang $Y$, Rong $X$, Luo $Y$, Li $P$, Han $Q$, Wei L, et al. A novel long non-coding RNA LINC00355 promotes proliferation of lung adenocarcinoma cells by down-regulating miR-195 and up-regulating the expression of CCNE1. Cel Signal. 2020;66:109462. https://doi.org/10.1016/j.cellsig.2019.109462 PMID: 31689506.

12. Lu S, Sun Z, Tang L, Chen L. LINC00355 promotes Tumor progression in HNSCC by hindering MicroRNA-195-mediated suppression of HOXA10 Expression. Mol Ther Nucleic Acids. 2020;19:61-71. https://doi.org/10.1016/j. omtn.2019.11.002 PMID: 31837606.

13. Wei GH, Wang X. IncRNA MEG3 inhibit proliferation and metastasis of gastric cancer via p53 signaling pathway. Eur Rev Med Pharmacol Sci. 2017; 21(17):3850-6 PMID: 28975980.

14. Hu Y, Ma Z, He Y, Liu W, Su Y, Tang Z. LncRNA-SNHG1 contributes to gastric cancer cell proliferation by regulating DNMT1. Biochem Biophys Res Commun. 2017;491(4):926-31. https://doi.org/10.1016/j.bbrc.2017.07.137 PMID: 28754593.

15. Renganathan A, Felley-Bosco E. Long noncoding RNAs in Cancer and therapeutic potential. Adv Exp Med Biol. 2017;1008:199-222. https://doi. org/10.1007/978-981-10-5203-3_7 PMID: 28815541.

16. Chen M, Wu X, Ma W, Zhou Q, Wang X, Zhang R, Wang J, Yang X. Decreased expression of IncRNA VPS9D1-AS1 in gastric cancer and its clinical significance. Cancer Biomark 2017; 21(1):23-28. DOl: https://doi.org/1 0.3233/CBM-170172. PMID: 29036784

17. Jiang T, Guo J, Hu Z, Zhao M, Gu Z, Miao S. Identification of Potential Prostate Cancer-Related Pseudogenes Based on Competitive Endogenous RNA Network Hypothesis. Med Sci Monit. 2018;24:4213-39. https://doi.org/1 0.12659/MSM.910886 PMID: 29923546.

18. Yan L, Wang P, Fang W, Liang C. Cancer-associated fibroblasts-derived exosomes-mediated transfer of LINC00355 regulates bladder cancer cell proliferation and invasion. Cell Biochem Funct. 2020;38(3):257-65. https:// doi.org/10.1002/cbf.3462 PMID: 31749189

19. Zhou F, Lei Y, Xu X, Zhou H, Liu H, Jiang J, et al. LINC00355:8 promotes cell proliferation and migration with invasion via the MiR-6777-3p/Wnt10b axis in hepatocellular carcinoma. J Cancer. 2020;11(19):5641-55. https://doi.org/1 0.7150/jca.43831 PMID: 32913459.

20. Kang W, Tong JH, Lung RW, Dong Y, Zhao J, Liang Q, et al. Targeting of YAP1 by microRNA-15a and microRNA-16-1 exerts tumor suppressor function in gastric adenocarcinoma. Mol Cancer. 2015;14(1):52. https://doi. org/10.1186/s12943-015-0323-3. PMID: 25743273.

21. Cui $Y$, Yang $Y$, Ren $L$, Yang J, Wang B, Xing T, et al. miR-15a-3p suppresses prostate Cancer cell proliferation and invasion by targeting SLC39A7 via Downregulating Wnt/beta-catenin signaling pathway. Cancer Biother Radiopharm. 2019;34(7):472-9. https://doi.org/10.1089/cbr.2018.2722 PMID: 31135177.

22. Zhao XQ, Tang H, Yang J, Gu XY, Wang SM, Ding Y. MicroRNA-15a-5p down-regulation inhibits cervical cancer by targeting TP53INP1 in vitro. Eur Rev Med Pharmacol Sci. 2019;23(19):8219-29. 31646552. https://doi.org/10.2 6355/eurrev_201910_19129.

23. Wu C, Zheng X, Li X, Fesler A, Hu W, Chen L, et al. Reduction of gastric cancer proliferation and invasion by miR-15a mediated suppression of Bmi1 translation. Oncotarget. 2016;7(12):14522-36. https://doi.org/10.18632/ oncotarget.7392 PMID: 26894855.

24. Zare A, Alipoor B, Omrani MD, Zali MR, Malekpour Alamdari N, Ghaedi H. Decreased miR-155-5p, miR-15a, and miR-186 Expression in Gastric Cancer Is Associated with Advanced Tumor Grade and Metastasis. Iran Biomed J. 2019;23(5):338-43 PMID: 31103022.
25. Bushati N, Cohen SM. microRNA functions. Annu Rev Cell Dev Biol. 2007; 23(1):175-205. https://doi.org/10.1146/annurev.cellbio.23.090506.123406. PMID: 17506695

26. Zhou X, Yang PC. MicroRNA: a small molecule with a big biological impact. Microrna. 2012;1(1):1 PMID: 25048083.

27. Di Leva G, Garofalo M, Croce CM. MicroRNAs in cancer. Annu Rev Pathol. 2014;9(1):287-314. https://doi.org/10.1146/annurev-pathol-012513-104715. PMID: 24079833.

28. van Mierlo G, Veenstra GJC, Vermeulen M, Marks $H$. The complexity of PRC2 subcomplexes. Trends Cell Biol. 2019;29(8):660-71. https://doi.org/10.1016/j. tcb.2019.05.004 PMID: 31178244

29. Kouznetsova VL, Tchekanov A, Li X, Yan X, Tsigelny IF. Polycomb repressive 2 complex-molecular mechanisms of function. Protein Sci. 2019;28(8):138799. https://doi.org/10.1002/pro.3647 PMID: 31095801.

30. Wang H, Xu P, Sun G, Lv J, Cao J, Xu Z. Downregulation of PHF19 inhibits cell growth and migration in gastric cancer. Scand J Gastroenterol. 2020; 55(6):1-7. https://doi.org/10.1080/00365521.2020.1766555. PMID: 32449434

31. Deng Q, Hou J, Feng L, Lv A, Ke X, Liang H, et al. PHF19 promotes the proliferation, migration, and chemosensitivity of glioblastoma to doxorubicin through modulation of the SIAH1/beta-catenin axis. Cell Death Dis. 2018;9(11):1049. https://doi.org/10.1038/s41419-018-1082-z PMID: 30323224

32. Tao F, Tian X, Ruan S, Shen M, Zhang Z. miR-211 sponges IncRNA MALAT1 to suppress tumor growth and progression through inhibiting PHF19 in ovarian carcinoma. FASEB J. 2018:fj201800495RR. https://doi.org/10.1096/fj.2 01800495 RR PMID: 29874124

33. Murakami H, Ito S, Tanaka H, Kondo E, Kodera Y, Nakanishi H. Establishment of new intraperitoneal paclitaxel-resistant gastric cancer cell lines and comprehensive gene expression analysis. Anticancer Res. 2013;33(10):4299307 PMID: 24122996.

\section{Publisher's Note}

Springer Nature remains neutral with regard to jurisdictional claims in published maps and institutional affiliations.
Ready to submit your research? Choose BMC and benefit from:

- fast, convenient online submission

- thorough peer review by experienced researchers in your field

- rapid publication on acceptance

- support for research data, including large and complex data types

- gold Open Access which fosters wider collaboration and increased citations

- maximum visibility for your research: over $100 \mathrm{M}$ website views per year

At BMC, research is always in progress.

Learn more biomedcentral.com/submissions 